\title{
A Sleep Monitoring System Based on Cloud Architecture
}

\author{
Z.H Li \\ College of Information Engineering \\ Qingdao University \\ China
}

\author{
Z.Q. Yu \\ College of Information Engineering \\ Qingdao University \\ China
}

\author{
W.L. Zhang \\ College of Information Engineering \\ Qingdao University \\ China
}

\begin{abstract}
Current sleep monitoring systems are often limited to sleep clinics and the patient may feel uncomfortable due to the numerous wired sensors attached to their body. Meanwhile most of existing works related to sleep quality rarely use questionnaire as a scoring tool. Even though several works have used questionnaires, users need to fill out the questionnaire every day. This paper proposes a novel sleep monitoring method using a smart sensor under the mattress without requiring users to wear special devices and fill out the whole questionnaire every day. The real-time data received from the sensor will be analysed and processed in the cloud server using an algorithm based on CSI. A client application is also developed to tell users how they slept and how to sleep better. Finally a set of experiments are conducted to verify the effectiveness and feasibility of the system. The result shows that accuracy of the system is more than 90 per cent.
\end{abstract}

Keyword-cloud architecture; sleep monitoring; CSI, questionnaire

\section{INTRODUCTION}

Sleep is a naturally recurring state of the higher vertebrates characterized by reduced response and consciousness to the external stimuli, relatively inhibited sensory activity, and inhibition of nearly all voluntary muscles[1]. Sleep, an important segment of the body, can make the brain have a good rest and integration. However, increasingly rapid pace of people's life gives them more and more pressure and results in sleep-deprived, aypnia and other sleep disorders. To monitor the sleep quality, many kinds of methods have been proposed[2, $3]$. While most of these methods use a wearable sensor, and a few works apply the scoring tools in the sleep monitoring. If we can monitor the sleep condition using unobtrusively sensor and evaluate the sleep quality of people according to the scoring tools, it will be helpful to improve people's sleep quality.

In this paper, we propose a cloud-based architecture for sleep monitoring. A PVDF piezoelectric film sensor is used to monitor the physiological signal of people to achieve the idea of non-invasive. The cloud server can collect the physiological signal monitored by smart sensor and analyse these data using a waveform method based on the Cubic Spline Interpolation
(CSI). The most important is that the results of the analysis can match with a scoring tool automatically to evaluate the sleep quality. The proposed system is also evaluated in a set of experiments to verify the effectiveness and feasibility.

The paper is organized as follows. Section 2 reviews the related work. Section 3 explains the architecture and design of the sleep monitoring system. Section 4 outlines the implementation and experimentation. Section 5 concludes the paper and provides the future work.

\section{RELATED WORK}

American Academy of Sleep Medicine(AASM) divides the sleep into three types, wake stage, rapid eye movement (REM sleep) and non-rapid eye movement (NREM sleep)[4].Among that NREM can also divide into shallow sleep and deep sleep. Kieran E. Brack insists that the changes of the sympathetic and vagus nerve in physiological reflection mechanism have some regularity during human different sleep stages, while heart rate and respiration are influenced obviously by the sympathetic and vagus nerve, therefore the variation trend of the heart rate and respiration in a whole night can largely reflect people's sleep information[5].

The Pittsburgh Sleep Quality Index (PSQI) is a most widely used questionnaire and scoring tool for long-term sleep quality evaluation[6]. On the basic of PSQI, Yin Bai proposes a questionnaire used to per-night sleep quality evaluation what we call the per-night questionnaire[7]. The subjects need to fill out the whole questionnaire manually every morning and they may not stick on it every day. Combining the sleep stage with the content of the per-night questionnaire, we develop a sleep monitoring system.

\section{ARCHITECTURE AND DESIGN OF THE SYSTEM}

The overall architecture of the proposed sleep monitoring system is depicted in Figure 1. The system has four basic modules, signal acquisition module, data transmission module, cloud server module and client display module. The cloud server is the most important module of this system. It includes three sub-modules, user management sub-module, data processing sub-module and data communication sub-module. 
The modular design and the cloud architecture features allow the system to be maintainable and robust. The major advantage of the system is that the sensor is covered with a bed sheet to conceal it and make it more comfortable to sleep on. Meanwhile the cloud server can receive the data of heart rate and respiratory signal from repeater in real-time, and fill out part of the contents automatically mentioned in the per-night questionnaire after specific analysis of these data.

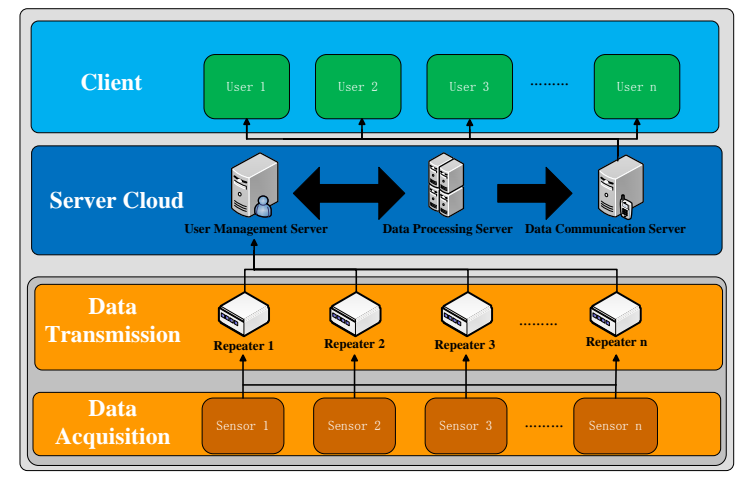

FIGURE I. THE OVERALL ARCHITECTURE

\section{A. Signal Acquisition Module}

The PVDF smart sensor under the mattress, which is developed by Shenzhen Seelen Technology, can detect heart rate, respiratory rate, body movement and other physiological signals continuously. A user can sleep comfortably without wearable sensors that may degrade the quality of sleep. The unobtrusive force sensor is particularly suitable for long-term use at home since the sensors are not expensive and no discomfort is caused to the user.

\section{B. Data Transmission Module}

To ensure that the sensor is unnoticeable and the system is easy to maintain, we use a repeater to transmit the data. The sensor picks up the heart rate, respiratory signal and body movement data, and the data are sent to repeater in structured form via RF. Then the repeater sends all data to the cloud server via $\mathrm{Wi}-\mathrm{Fi}$.

\section{Data Storage/Analysis and Communication Module}

This module includes threes sub-modules:

The function of the first sub-module is user management. A database management sub-system (MySQL) is used to store the users' information and the raw data received continuously from the repeater. A website has been developed using LAMP (Linux, Apache, MySQL and PHP). Users should register first and fill out the basic information, including a portion of the per-night questionnaire, because these parts cannot be inferred by the heart rate and respiratory signal.

The function of the second sub-module is data processing. The analysis results of the data will be stored in database. The signal processing algorithm used in the system is waveform method based on the CSI matching envelope, which is proposed and tested in this paper. CSI algorithm is widely used in many domains, especially in biomedical signals processing[8, 9]. The basic idea of our algorithm can be described as follows:
Step1: Given a heart rate signal ${ }^{x 1(t)}$, identify all extremes of $x 1(t)$.

Step2: Generate the upper envelope $U(t)$ and the lower envelope $L(t)$ respectively by CSI among all extremes (the maxima and the minima).

Step3: Compute the mean of the two envelopes $m(t)=(U(t)+L(t)) / 2$.

Step4: Find the peak of $L(t)$, and the number of peaks are the heart rate.

Given a respiratory signal $x 2(t)$, we can also use the above method. An artificial-intelligence sleep auto staging algorithm, which is proposed by Wang Chenlei [10], is used to infer the result of the sleep stage according to the heart rate and the respiratory rate. From the result we can obtain the time of the wake stage, the shallow sleep stage, the deep sleep stage and the REM stage respectively corresponding to the part content of the per-night questionnaire. The question of 1-5 can be filled out automatically, while others need to be filled out when the users registered for the first time. Finally the system can generate an integrated result of the per-night questionnaire and calculate the user's sleep quality score according to the scoring scheme shown in paper [7].

The function of the third sub-module is data communication. A report of the sleep quality is sent to the application of user's client in the morning.

\section{Client Display Module}

To make the users obtain their sleep status, a feedback of user's sleep quality and other information will be showed in the application of the phone or the pad. The users can obtain a report of their sleep quality every morning.

\section{IMPLEMENTATION AND EXPERIMENTATION}

At the first phase of the system, we develop and test the above proposed modules. After registration in the website, users' sensor and repeater can bind to their account. The acquired data of the heart rate and the respiratory signal are generated in special format, which enables the sending of the data relatively quick and compact. The data protocol is proprietary in our system. With this format, it is easy to parse in the server. After collection and analysis of the data, the cloud server can show the sleep result of last night in the website. Users can login by entering the name and password to know their sleep condition. The sleep condition of one subject is showed in Figure 2. 


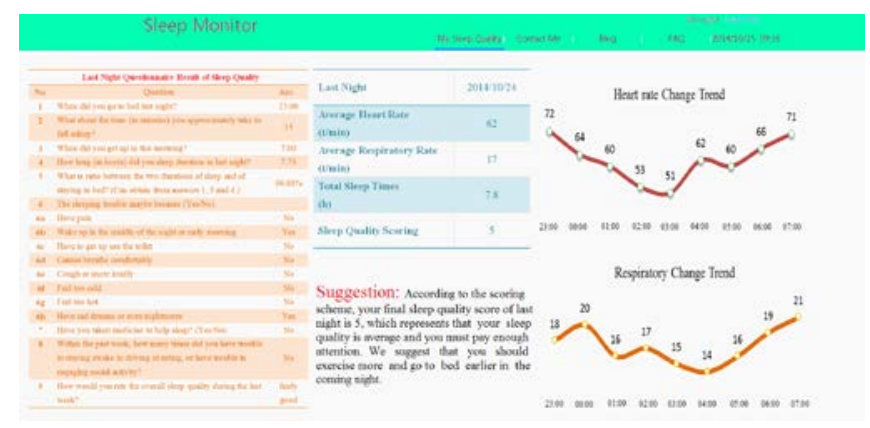

FIGURE II. HE SERVER WEBSITE

We also conduct a set of experiments to evaluate the performance of our system that involves 6 subjects and total 7 nights of sleep. During the night, all the subjects go to bed at 23:00with the smart sensor under their mattress, and they also need to fill out the per-night questionnaire manually in the morning. Meanwhile, our system can generate a per-night questionnaire automatically. In the seventh day, we compare the two questionnaires and calculate a matching accuracy. The results show in Table 1 indicates that the accuracy of our system is more than 90 per cent. AHR1 and ARR1 mean the average heart rate and the average respiratory rate respectively which are measured by our system. While the AHR2 and the ARR2 mean the actual heart rate and the actual respiratory rate of the subjects, respectively.

TABLE I. PERFORMANCE OF THE SYSTEM ASSESSMENT

\begin{tabular}{|c|c|c|c|c|c|}
\hline Subjects & AHR1 & AHR2 & ARR1 & ARR2 & Matching accuracy \\
\hline $\mathbf{1}$ & 62 & 64 & 17 & 15 & $95.0 \%$ \\
\hline $\mathbf{2}$ & 65 & 63 & 16 & 15 & $96.2 \%$ \\
\hline $\mathbf{3}$ & 68 & 65 & 15 & 16 & $93.6 \%$ \\
\hline $\mathbf{4}$ & 66 & 67 & 14 & 13 & $96.3 \%$ \\
\hline $\mathbf{5}$ & 61 & 65 & 15 & 15 & $91.1 \%$ \\
\hline $\mathbf{6}$ & 67 & 66 & 14 & 15 & $95.7 \%$ \\
\hline
\end{tabular}

An analysis result of the sleep quality is sent to the client. The user can obtain their sleep quality, the average heart rate, the average respiratory and the total sleep times of last night. We will continue to develop additional functionality. The client application is showed in Figure 3.

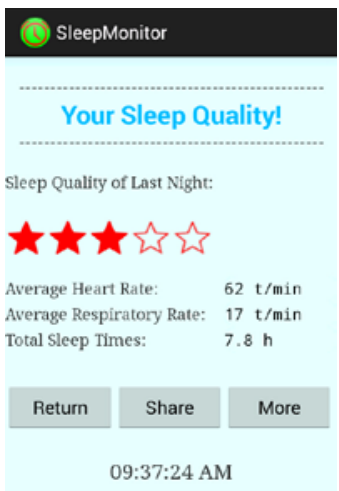

FIGURE III. THE CLIENT DISPLAY

\section{CONCLUSIONS}

In this paper, we design a cloud-based architecture for the sleep monitoring. Several features such as heart rate, respiratory and body movements are acquired from a smart sensor, and an algorithm based on CSI is proposed to infer the heart rate and respiratory rate. After filled out the content of the per-night questionnaire, system can generate a report about user's sleep quality. The user can check out the report from the app or the website. The experimental result shows that the accuracy of the system is more than 90 per cent. The future works envisaged include designing a more useful questionnaire according to PSQI, and filling out more contents of the questionnaire automatically. The influence of the light and noise will be considered to analyse the sleep quality of people.

\section{ACKNOWLEDGEMENT}

The research work was supported by National Development and Innovation Committee under Item Community Health Service Oriented Digital Medical Cloud Service Platform Construction.

\section{REFERENCES}

[1] Sleep - Wikipedia, the free encyclopedia. http://en.wikipedia.org/wiki/Sleep.

[2] Takuji Suzuki, Kazushige Ouchi and Ken-ichi Kameyama and Masaya Takahashi, Development of a Sleep Monitoring System with Wearable Vital Sensor for Home Use.BIODEVICES,pp.326-331,2009.

[3] Jin U. Bak, Nikolaos Giakoumidis, Gagyung Kim, Haiwei Dong and Nikolaos Mavridis, An Intelligent Sensing System for Sleep Motion and Stage Analysis. Procedia Engineering, Volume 41, pp.1128-1134, 2012.

[4] Michael H. Silber, Sonia Ancoli-Israel, Michael H. Bonnet, Sudhansu Chokroverty, The visual scoring of sleep in adults. Journal of Clinical sleep Medicine, 3(2), pp.121-13,2007.

[5] Kieran E. Brack, John H. Coote\& G André Ng, Interaction between direct sympathetic and vagus nerve stimulation on heart rate in the isolated rabbit heart. Experimental Physiology. 89(1), pp.128-139,2004.

[6] Daniel J. Buysse, Charles F. Reynolds, Timothy H. Monk, Susan R. Berman and David J. Kupfer, The pittsburgh sleep quality index: a new instrument for psychiatric practice and research. Psychiatry Research, 28(2), pp.193-213,1989.

[7] YinBai, BinXu, Yuanchao Ma, Guodong Sun and Yu Zhao,Will you have a good sleep tonight? Sleep quality prediction with mobile phone. BodyNets, pp.124-130,2012.

[8] Norden E. Huang, Zheng Shen, Steven R. Long, Manli C. Wu, Hsing H. Shih, Quanan Zheng, Nai-Chyuan Yen, Chi Chao Tung and Henry H. Liu, The empirical mode decomposition and Hilbert spectrum for nonlinear and non-stationary time series analysis. The Royal Society, pp.903-995,1998.

[9] Nai-Fu Chang, Cheng-Yi Chiang, Tung-Chien Chen and Liang-Gee Chen, Cubic Spline Interpolation with Overlapped Window and Data Reuse for On-line Hilbert Huang Transform Biomedical Microprocessor. Annual International Conference of the IEEE Engineering in Medicine and Biology Society, pp.7091-7094,2011.

[10] Chenlei Wang, Design and Implementation of a Sleep Monitor System Based on DSP. South China University of Technology,2013. 